\title{
Assessment of the risk of African swine fever introduction into Finland using NORA - a rapid tool for semiquantitative assessment of the risk
}

\author{
J. Kyyrö | L. Sahlström ｜ T. Lyytikäinen
}

Risk Assessment Research Unit, Research and Laboratory Department, Finnish Food Safety Authority Evira, Helsinki, Finland

Correspondence

T. Lyytikäinen, Finnish Food Safety Authority Evira, Helsinki, Finland.

Email: tapani.lyytikainen@evira.fi

\begin{abstract}
Summary
The NORA rapid risk assessment tool was developed for situations where there is a change in the disease status of easily transmissible animal diseases in neighbouring countries or in countries with significant interactions with Finland. The goal was to develop a tool that is quick to use and will provide consistent results to support risk management decisions. The model contains 63 questions that define the potential for entry and exposure by nine different pathways. The magnitude of the consequences is defined by 23 statements. The weight of different pathways is defined according to the properties of the assessed disease. The model was built as an Excel spreadsheet and is intended for use by animal health control administrators. As an outcome, the model gives the possible pathways of disease entry into the country, an overall approximation for the probability of entry and the subsequent exposure, an overall estimate for the consequences and a combined overall risk estimate (probability multiplied by magnitude of consequences). Model validity was assessed by expert panels. Outside Africa, African swine fever is currently established in Russia and Sardinia. In addition, there have been cases in both wild boar and domestic pigs in Latvia, Lithuania, Poland and Estonia. Finland has frequent contacts with Russia and Estonia, especially through passengers. The risk of African swine fever (ASF) introduction into Finland was tested with NORA for the situation in December 2015, when ASF was endemic in many parts of Russia, Africa and Sardinia and was present in Baltic countries and in Poland. African swine fever was assessed to have a high probability of entry into Finland, with high consequences and therefore a high overall risk.
\end{abstract}

\section{KEYWORDS}

African swine fever, risk assessment, rapid tool, qualitative, disease introduction

\section{1 | INTRODUCTION}

Risk assessment can be either quantitative or qualitative, with both types being equally valid (Dufour et al., 2011). Risk assessment is a good method to aid risk managers, for example with questions of emerging risks. However, the downside is often the time requirement of the method. There is often a need for information "as soon as possible", but the scientific quantitative risk assessment procedure may take months or even years. Therefore, various qualitative or semiquantitative methods have been developed to address risk questions in a matter of days. According to Wilson and Crouch (2001), risk can be defined as a combination of the probability of an adverse event occurring and the severity of the occurrence, giving: Risk $=$ Probability $\times$ Severity . 
Rapid risk assessments are normally used when there is a need for a rapid and rough estimate of a risk shortly after it emerges to allow urgent risk management actions. Compared to a thorough scientific risk assessment, rapid risk assessment tools are less reliable and less accurate. There is often a need for expert panels instead of scientific evidence. Rapid risk assessments can be complex and demanding due to the tight time schedule and limited information available in rapidly changing situations.

The questions relating to the risk are answered and added together to gain an overall value, which is then usually converted to a four- to nine-level verbal scale (Dufour et al., 2011; Moutou, Dufour, \& Ivanov, 2001). The EFSA scientific opinion on risk assessment terminology (EFSA, 2012) provides an overview of categories used for different levels of risk. Words used for categories vary and, e.g., Mur, Martínez-López, and Sánchez-Vizcaíno (2012) used a sixlevel scale (negligible, very low, low, medium, high and very high) when assessing the risk of African swine fever introduction into the EU through transport-associated routes. Rapid risk assessment tools are normally based on a flow chart (Morgan, Kirkbride, Hewitt, Said, \& Walsh, 2009; Palmer, Brown, \& Morgan, 2005) or a risk matrix (ECDC, 2011). A flow chart is a structured way to attain an end point by answering a series of linked questions, while a matrix is a way of combining two categorized qualitative estimates (e.g., probability and consequence). Defra's International Disease Monitoring Veterinary Science Team has developed a tool to give a rapid semiquantitative measure of the risk of disease introduction (Roberts, Carbon, Hartley, \& Sabirovic, 2011). It uses a decision matrix based on the hypothesis that a disease could be introduced into the UK through trade (legal and illegal) in live animals or animal products, via transport and fomites or through the movement of vectors or wildlife.

Morgan et al. (2009) developed a rapid, systematic, objective and transparent method for assessing the risk to the UK population from new and emerging infections arising anywhere in the world. They assumed the method to provide a means of communicating risk in a systematic manner to stakeholders and to provide an audit trail for the decisions made. The method was based on two flow charts, one for the probability and one for the impact of the risk. They published both the probability and impact of Chikungunya as being minimal when assessed with their tool. However, their tool did not combine the probability and impact.

The evaluation of risk assessment based on systematic documentation provides an important means of identifying where improvements can be made, as well as an evidence base for future risk assessments and responses to events (WHO, 2012). According to WHO (2012), rapid risk management of acute public health events reduces or prevents disease in affected populations and reduces negative social and economic consequences. Additional benefits are defensible decision-making, the implementation of appropriate and timely control measures, more effective operational communication, more effective risk communication and improved preparedness.

The reason for us to develop a specific tool for Finland is based on the special nature of our country: the risks are different from those in the majority of other EU countries. We are free from many common diseases, our country is isolated in many ways, the trade in animals is minimal, and native data are readily available.

African swine fever (ASF) is endemic in sub-Saharan Africa, Madagascar, and since 1978 also in Sardinia (EFSA AHAW Panel, 2010; Sánchez-Vizcaíno, Mur, \& Martínez-López, 2013). More recently, African swine fever virus (ASFV) was introduced in Georgia in 2007, probably through the feeding of pigs with imported infected meat products or garbage. Since then, ASFV has spread markedly in the Caucasus area (Georgia, the Russian Federation, Azerbaijan, Armenia, Ukraine and Belarus), causing many outbreaks in domestic backyard or industrially kept pigs and/or wild boar populations (EFSA AHAW Panel, 2010, 2014; Gogin, Gerasimov, Malogolovkin, \& Kolbasov, 2013; Sánchez-Vizcaíno et al., 2013). The situation substantially worsened when the disease entered the European Union in the Baltic countries at the end of 2013-early 2014. Several outbreaks within Lithuania and Poland were reported to OIE (EFSA AHAW Panel, 2014). The genotype currently causing outbreaks in Lithuania, Poland and also very widely in Estonia and Latvia (OIE, 2016; Sánchez-Vizcaíno, Mur, Gomez-Villamandos, \& Carrasco, 2015; Sánchez-Vizcaíno, Mur, Sánchez-Matamoros, \& Martínez-López, 2014) is highly virulent (FAO, 2013; Gogin et al., 2013). Recently, several ASF risk assessments on European level have been conducted and those have used a qualitative approach (EFSA AHAW Panel 2010, 2014; FAO, 2013), semiquantitative tools (De la Torre et al., 2013; Mur et al., 2012) or quantitative models (Mur et al., 2012).

Our aim was to develop a rapid, systematic and transparent risk assessment method to assess the risk of animal diseases spreading into Finland and to use it for ASF assessment, as the disease has spread alarmingly in Russia and in the Baltic countries.

\section{2 | MATERIALS AND METHODS}

NORA was built as an Excel spreadsheet, and its use does not require advanced computer skills. The method used is principally based on the risk assessment method developed by the World Organization for Animal Health (OIE) (2011).

This method runs through three different steps: (i) release and exposure, (ii) consequences and (iii) overall risk estimation. The first step, release and exposure assessment, is an estimation of the likelihood of a hazard being introduced into Finland and a susceptible animal becoming exposed to the hazard. This can be referred to as the probability of occurrence. Secondly, consequences are assessed, including economic and health consequences. The third step is risk estimation, which combines the results of the two preceding steps.

\section{1 | Identification of relevant pathways}

Nine different pathways have been identified for the entry and release of a disease: with live animals (i), sperm and embryos (ii), products of animal origin (iii), passengers (iv), wildlife (v), air currents 
and/or vectors (vi), animal transport vehicles (vii), feed and bedding materials (viii) and other goods and traffic (ix) (Figure 1).

There are two sets of questions for each pathway. Every question comes with guidance on things to consider when answering. Also, many multiple choice answers come with definitions of choices. The first set of questions defines the possibility of a pathway for the scenario in question (Table 1). If the pathway is relevant, the other set of questions defines the probability of that pathway in the scenario (Table 2). If the pathway is not relevant, one moves on to the questions of the following pathway. These pathways are only relevant if the entry of the disease can be followed by the exposure of a susceptible animal.

\section{2 | Probabilities of pathways}

The probability of a pathway is a product of the questions defining the possibility and the magnitude of certain actions that are risk related. The magnitude questions scale the likelihood of an event by means of multiple choice (three choices). The answers may either decrease or increase the risk. The risk may be increased if the answer is "yes" to a risk-increasing question such as: "Has there been import of animals from the area known to be infected during the past year?" An example of a risk-decreasing question is the following: "Is adequate import quarantine in use?"

The questions concerning probabilities are given a point estimate between 0 and 1 . Pathways may be made up of more than one entity. The estimates for the questions regarding the same pathway are then multiplied with each other within an entity of questions. The multiplied scores of entities are added together and divided by the number of entities. An example of an entity is the import of live animals, and another is the smuggling of live animals in the pathway "with live animals".

\subsection{Combining the probabilities of the pathways}

NORA includes different weights for entry pathways (Table 3) and for the subfactors of consequences (Table 5). Different pathogens transmit in different ways; e.g., some pathogens can spread only through direct contact and some may spread with, e.g., air current and vectors. Therefore, the entry pathways need to be weighted depending on the transmission properties of the pathogen. There are three different weighting alternatives in NORA depending on the transmission of the disease. One is for easily transmitted viral diseases that can also be transmitted via animal products (African swine fever and classical swine fever), one is for easily transmitted viral diseases with an airborne transmission feature (Bluetongue), and one is for diseases with both properties (foot-and-mouth disease). The weighting factors were assigned as follows: firstly, the nine different pathways were ranked so that the pathways with the same significance were given the same rank. Then, 100 points were divided between the pathways according to their ranks. The probabilities of different pathways are added together and then divided by the total weights for the pathways (i.e., 100).

\subsection{Defining the consequences of a disease}

The list of statements for the consequences of a hazard (Table 4) follows the questions for the pathways of entry. These are yes/no
FIGURE 1 Possible pathways for the entry of diseases into Finland. [Colour figure can be viewed at wileyonlinelibrary.com]

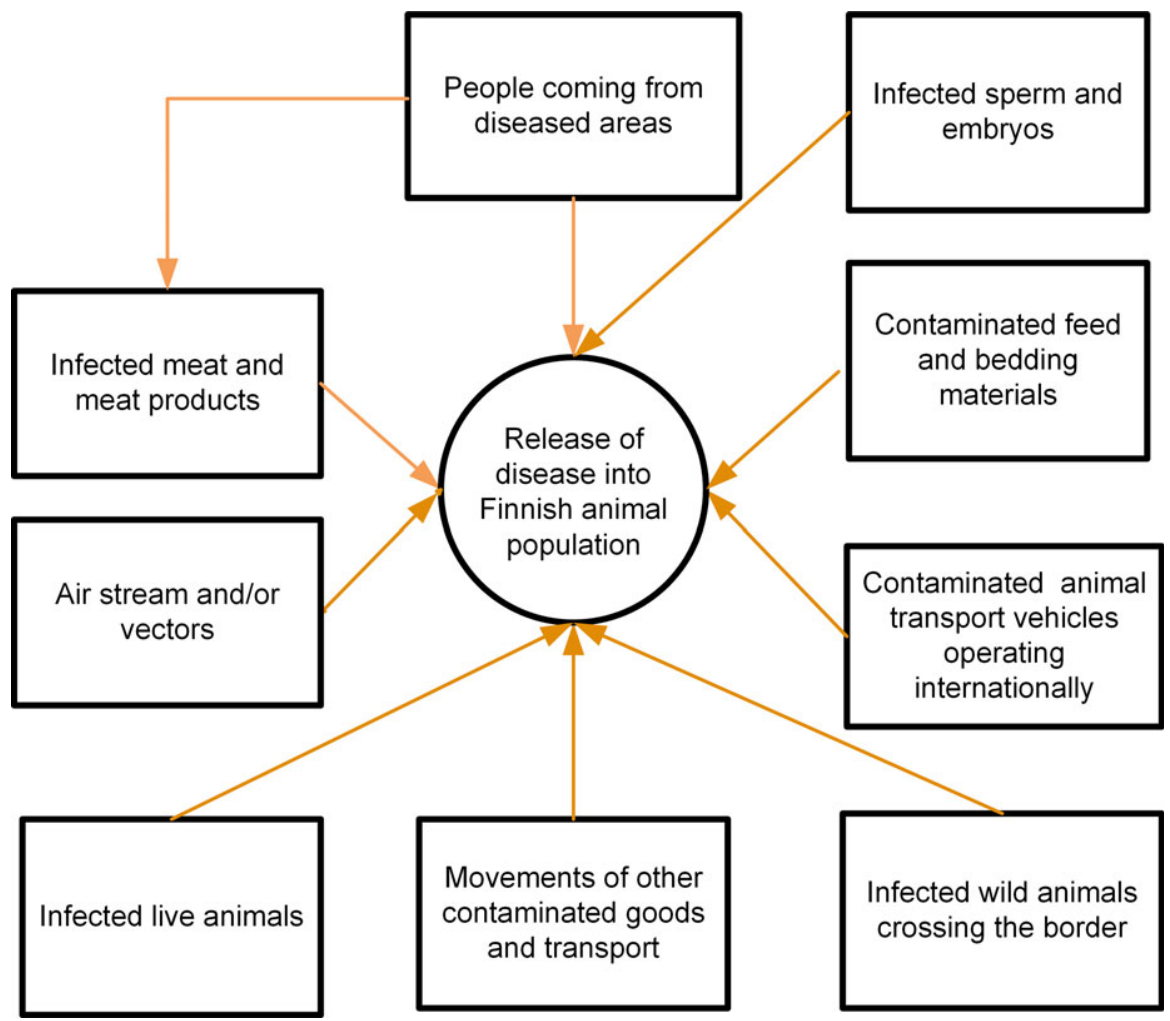


TABLE 1 Questions defining whether a pathway is relevant

\section{With live animals}

\section{Is it possible for the disease to spread with live animals?}

Is there import of live animals from the country(ies) in question?

Is it possible for these imported animals to come into contact with susceptible animals?

2. With sperm and embryos

Is it possible for the disease to spread with gametes?

Is there import of gametes from the country(ies) in question?

Is it possible for these imported gametes to end up in contact with susceptible animals?

3. With products of animal origin

Is it possible for the disease to spread with products of animal origin?

Is there import of products of animal origin that may spread the disease from the country(ies) in question?

Is it possible for farmers or farm workers to bring these products with them?

Is it possible for the pathogen in these products to be carried with people to susceptible animals in Finland?

4. With passengers

Is it possible for the disease to be spread with humans?

Is it possible for a traveller in the country(ies) in question to have been in contact with infected animals or materials?

Is it possible for a person who has been in an animal facility in the country(ies) in question to come into contact with susceptible animals in Finland?

Is it possible for this person to enter an animal facility in Finland within the infectious period?

\section{With wildlife}

Is it possible for the disease to infect wildlife?

Is it possible for infected wildlife to migrate to Finland?

Is it possible for infected wildlife or their secretions to come into contact with susceptible animals in Finland?

6. With air currents and/or vectors

Is it possible for the disease to spread with air currents or vectors?

Is it possible for the disease to be transmitted to Finland with air currents or vectors?

Is the climate currently suitable for spread with air currents or vectors?

Is it possible at the moment for disease spread with air currents or vectors to come into contact with susceptible animals in Finland?

7. With animal transport vehicles

Is it possible for the disease to spread with an animal transport vehicle?

Are there animal transport vehicles coming to Finland from the country(ies) in question?

Is it possible for an animal transport vehicle to come into contact with susceptible animals in Finland while infectious?

8. With feed or bedding materials

Is it possible for the disease to spread with feed or bedding materials?

Is there import of animal feed or bedding materials from the country(ies) in question?

Is it possible for the feed or bedding materials to come into contact with susceptible animals in Finland while infectious?

9. With other goods or traffic

Is it possible for the disease to spread with other goods and traffic?

Are other goods or land traffic coming from the country(ies) in question?

Is it possible for these goods or traffic to come into contact with susceptible animals in Finland while infectious?

questions, and they can only increase the risk estimate for consequences. Statements for the value of the animal sector define how many species of animals the disease in question is capable of affecting. The foodstuff values of the affected sectors are combined. Animal species taken into account in NORA are: cattle, poultry, pigs, cultivated fish, deer, reindeer, wild fish, hares, sheep and wild fowl.

The second set of statements defines the disease's classification in the national legislation. Diseases are divided according to their importance for the national economy, which thus defines the importance of their control.

The third set of statements concerns the multiplier effects of disease introduction. The classification of the disease, the multiplier effects of its entry and the menace of the disease together form an additive entity called the severity of the disease.

The weighting factors for the consequences are the same for all the assessed diseases (Table 5). The highest factor is for the impact on the 
TABLE 2 Questions defining the probability of a pathway

\section{With live animals}

How many batches of animals have been imported during the past year from the country(ies) in question?

Has there been import of animals from the area known to be infected during the past year?

Is the health status of the importing farm(s) known and acceptable?

Is the health status of the imported animals known and acceptable?

Is adequate import quarantine in use?

Is it possible that the smuggling of live animals from the country(ies) in question is significant?

Is it known that a significant number of sensitive animals have been imported against the rules from the country(ies) in question?

\section{With gametes}

How many batches of gametes have been imported from the country(ies) in question during the past year?

Has there been import of gametes from the area known to be infected during the past year?

Is the health status of the importing farm(s) known and acceptable?

Is the health status of the imported gametes known and acceptable?

Is it possible that the smuggling of gametes from the country(ies) in question is significant?

Is it known that a significant quantity of gametes has been imported against the rules from the country(ies) in question?

3. With products of animal origin

Is it possible that products of animal origin from the infected areas are brought for sale in Finland?

How large a quantity of products of animal origin from the country(ies) in question is sold in Finland?

How many of these products remain infective until in possession of the end user?

How many passengers come from the country(ies) in question?

How many citizens of the country(ies) in question work on Finnish farms?

Is it possible that the smuggling of products of animal origin from the country(ies) in question is significant?

Is it known that a significant quantity of products of animal origin has been imported against the rules from the country(ies) in question?

4. With passengers

Do the professionals working with animals (shearers, veterinarians, advisors) in the country(ies) in question come to Finland?

How many citizens of the country(ies) in question work on farms in Finland?

How many passengers come from the country(ies) in question?

5. With wildlife

How much wildlife can migrate to Finland while infectious from the country(ies) in question?

How much are the wild animals in contact with sensitive animals in Finland?

6. With air currents and/or vectors

How far is the infected area from the Finnish border?

How many sensitive animals are there in the area where the pathogen may end up in Finland?

7. With animal transport vehicles

How long may the pathogen remain infectious outside an animal?

How many infectious animal transport vehicles come from the infected area to Finland?

8. With feed or bedding materials

How long may the pathogen remain infectious outside an animal?

How many batches of feed or bedding materials have come to Finland from the infected area during the past year?

9. With other goods or traffic

How long may the pathogen remain infectious outside an animal?

How large a quantity of infectious other goods or traffic has come to Finland during the past year?

export of foodstuffs, the second highest is for the classification in the legislation, the third is for other multiplier effects, the fourth is for the menace and zoonotic features of the disease with identical weights, and the smallest factor is for the economic value of the animal sector(s) affected. These weighting factors rely on the fact that NORA is constructed for use in the administration of animal health control.
The consequences of the hazard are counted accordingly: the summed animal sector values are multiplied by the first three multiplier effects and added to the last multiplier effect ("the disease-free status is lost and its restoration demands significant resources") and to the disease classification in the legislation, menace and the possible zoonotic nature of the disease. 


\subsection{Combining probability and consequences}

The estimate for probability is multiplied by the estimate for the consequences to calculate the total risk score. The probability and magnitude of the consequences and the total scores can then be transformed into a five-level categorical scale: negligible, low, moderate, high or very high risk (Tables 6, 7 and 8). The verbal scores are explained in Table 9.

The equation for the combined overall risk:
Initially, risk assessments for ASF introduction were performed with NORA by the research group. Later on, expert elicitation groups were formed to validate the questions concerning probabilities of pathways for ASF introduction. The first was a preliminary test with four experts at Evira that took place in June-July 2015. Three workshops were then held for experts in December 2015: one for veterinarians specializing in transmissible animal diseases (five persons), one for postgraduates in veterinary medicine (nine persons) and the final one for experts of animal health risk management at Evira (five persons). In February 2016, we conducted one more workshop for

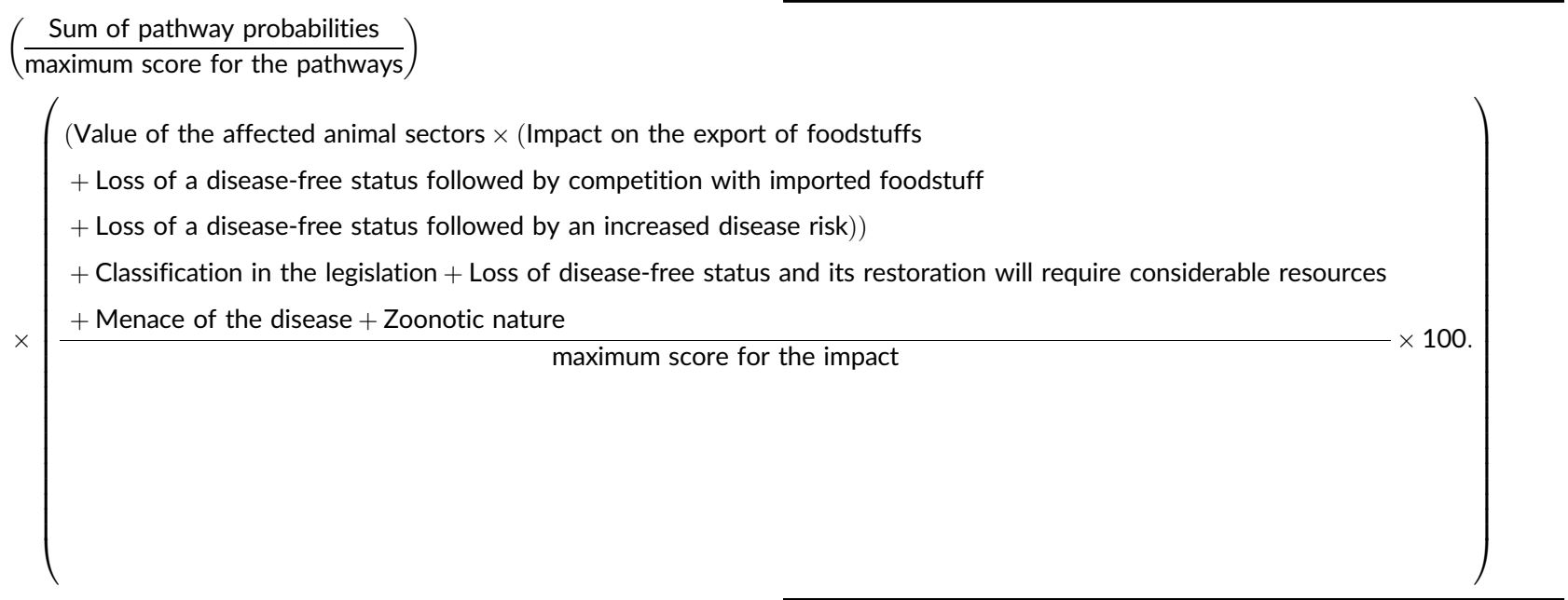

\subsection{Validation of the tool}

We first conducted a preliminary review of rapid risk assessment tools in May 2012, followed by a questionnaire to the five experts in charge of animal health issues at the Finnish Food Safety Authority Evira and two experts in the Ministry of Agriculture and Forestry. The replies to the questionnaire were discussed in a meeting in November 2012. In August 2013, a follow-up meeting was held with the five experts from Evira and another meeting with three experts in January 2014.

TABLE 3 Weights for different entry and exposure pathways for African swine fever (ASF), classical swine fever (CSF), Bluetongue (BT) and foot-and-mouth disease (FMD)

\begin{tabular}{lrrr|}
$\begin{array}{l}\text { Weighting factors for the } \\
\text { probability of the pathways }\end{array}$ & ASF, CSF & BT & FMD \\
\hline With animals & 30 & 35 & 30 \\
\hline With sperm and embryos & 15 & 17 & 13 \\
\hline With animal products & 15 & 0 & 13 \\
\hline With passengers & 5 & 0 & 4 \\
\hline With wildlife & 15 & 12 & 13 \\
\hline With air currents and/or vectors & 0 & 30 & 13 \\
\hline With animal transport vehicles & 10 & 6 & 6 \\
\hline With feed or bedding & 5 & 0 & 4 \\
\hline With other goods or traffic & 5 & 0 & 4 \\
\hline Sum & 100 & 100 & 100 \\
\hline
\end{tabular}

risk assessors at Evira (12 persons) working with plant health- and food-related risks.

The four validation groups (veterinarians specializing in transmissible animal diseases, postgraduates in veterinary medicine, experts of animal health risk management at Evira and risk assessors at Evira working with plant health- and food-related risks) answered the questions concerning the pathways (Identification of relevant pathways, Table 1) and their probabilities (Probabilities of pathways, Table 2). Weights of pathways (Table 3), weights for different consequence factors (Table 5) and the answers regarding the list of statements for the consequences of a disease (Table 4) were given by the research group and not changed by the validation groups. The results for overall pathways probabilities are presented separately for each validation group and also as a mean across all the validation groups.

\section{3 | RESULTS}

\section{1 | Probability of the risk}

The outcomes of the validation groups were mostly consistent: all groups (but not all individuals) assessed all the routes of entry and exposure to be relevant, except for air currents and/or vectors (Figure 1). Moreover, they evaluated the probability of African swine fever being released into the Finnish pig population to be high $(0.18$ and 0.16 ) or very high (0.21 and 0.23 ), respectively, with the mean 
TAB LE 4 List of statements for consequences

\begin{tabular}{|c|c|}
\hline & $\begin{array}{l}\text { Score for } \\
\text { each option }\end{array}$ \\
\hline \multicolumn{2}{|l|}{ 1. Value of the animal sector as a foodstuff } \\
\hline The disease can spread to cattle & 69.69 \\
\hline The disease can spread to horses & 0.08 \\
\hline The disease can spread to poultry & 12.99 \\
\hline The disease can spread to pigs & 10.06 \\
\hline The disease can spread to cultivated fish & 2.83 \\
\hline The disease can spread to deer & 2.62 \\
\hline The disease can spread to reindeer & 0.63 \\
\hline The disease can spread to wild fish & 0.46 \\
\hline The disease can spread to hares & 0.40 \\
\hline The disease can spread to sheep & 0.15 \\
\hline The disease can spread to wild fowl & 0.08 \\
\hline \multicolumn{2}{|l|}{ 2. Disease classification in the legislation } \\
\hline Dangerous animal disease & 24 \\
\hline Easily transmissible animal disease & 32 \\
\hline Controlled animal disease & 16 \\
\hline Notifiable animal disease & 8 \\
\hline $\begin{array}{l}\text { A new disease or form of the disease not } \\
\text { mentioned in the legislation }\end{array}$ & 20 \\
\hline Other animal disease & 0 \\
\hline \multicolumn{2}{|l|}{$\begin{array}{l}\text { 3. There are significant multiplier effects for } \\
\text { the entry of the disease }\end{array}$} \\
\hline Significant impact on the export of foodstuffs & 50 \\
\hline $\begin{array}{l}\text { The disease-free status is lost and the import } \\
\text { of foodstuffs causes additional competition } \\
\text { and significantly endangers domestic production }\end{array}$ & 10 \\
\hline $\begin{array}{l}\text { The disease-free status is lost and it raises the } \\
\text { overall disease risk }\end{array}$ & 10 \\
\hline $\begin{array}{l}\text { The disease-free status is lost and its restoration } \\
\text { demands significant resources }\end{array}$ & 30 \\
\hline \multicolumn{2}{|l|}{ 4. Menace of the disease } \\
\hline Public opinion sees the disease as menacing & 100 \\
\hline \multicolumn{2}{|l|}{ 5. Zoonotic nature } \\
\hline $\begin{array}{l}\text { The disease is a significant zoonosis } \\
\text { (causes serious symptoms in } \\
\text { people and/or spreads easily within } \\
\text { the human population) }\end{array}$ & 100 \\
\hline
\end{tabular}

being high (0.195) (Figure 2, Table 6). To study the differences between the groups, we examined the mean ranks of probabilities with the Kruskal-Wallis test, because the estimates within the groups were not normally distributed and the numbers of respondents per group were low. The Kruskal-Wallis test revealed no significant difference $(p=.309)$ between the mean ranks of probabilities for these groups (Figure 2).

Even though the mean overall probability estimate was quite similar across the four groups of respondents, the scores for the nine different pathways varied somewhat (Figure 3). The mean overall scores (probability $\times$ weight) across the validation groups were as follows: the highest score was 6.7 for products of animal origin, 3.6 for live animals and for passengers, 1.8 for feed and bedding materials, 1.2 for other goods and traffic, 1.1 for sperm and embryos and for animal transport vehicles, and the lowest score 0.45 for wild boar.

To study the within-group variation in probabilities between the groups, we examined the mean ranks of residuals with the KruskalWallis test. The variation differed significantly between the groups for the following routes: with sperm and embryos $(p=.013)$, with products of animal origin ( $p=.035$ ), with passengers $(p=.001)$, with wildlife $(p=.000)$, with animal transport vehicles $(p=.000)$ and with other goods and traffic $(p=.002)$. The variation in the overall probability also differed significantly ( $p=.013$ ) between the groups.

To determine how many respondents are needed to derive a solid estimate and as little variation as possible, we simulated the mean and $95 \%$ confidence interval for the 31 respondents in four groups of respondents with a Monte Carlo simulation model (Figure 4). The specialists' estimates varied the least, and those of the plant health and food safety risk assessors varied the most. Therefore, the number of respondents needed to provide a solid estimate strongly depends on the background of the respondent.

\subsection{Consequences of the risk}

The consequences of ASF release into Finland (Table 5) were evaluated by the research group to be 17.36 representing a high impact on the verbal scale (Table 7). We did not evaluate the consequences in the validation groups, as the consequence claims are straightforward and do not include personal opinions.

To test the consistency of the tool, four diseases were tested with NORA for their consequences: Schmallenberg virus,

TABLE 5 Weights for different consequence factors and indices for ASF

\begin{tabular}{|c|c|c|c|}
\hline Animal sector affected & 0.1 & $10.06 \times 0.1=1.006$ & 10 \\
\hline Multiplier effect of exposure 1 (export) & 100 & $50 \times 100=5,000$ & 5,000 \\
\hline Multiplier effects of exposure $2,3,4$ & 50 & $50 \times 50=2,500$ & 2,500 \\
\hline Zoonotic feature of the disease & 11 & 0 & 1,100 \\
\hline
\end{tabular}


TABLE 6 Converting the numerical value of the probability into a verbal score

\begin{tabular}{|ll}
\hline Probability of the risk & \\
\hline Numerical values & Verbal score \\
$<0.01$ & Negligible \\
$0.01-0.024$ & Low \\
\hline $0.025-0.049$ & Moderate \\
$0.05-0.199$ & High \\
\hline $0.2-1$ & Very high \\
\hline
\end{tabular}

TABLE 7 Converting the numerical value of the consequences into a verbal score

\begin{tabular}{ll}
\hline Consequence claims & \\
\hline Numerical values & Verbal score \\
\hline$<1$ & Negligible \\
\hline $1-5$ & Low \\
\hline $5-15$ & Moderate \\
\hline $15-50$ & High \\
\hline $50-100$ & Very high \\
\hline
\end{tabular}

TABLE 8 Converting the numerical value of the overall risk into a verbal score

\begin{tabular}{ll}
\hline Overall risk score & \\
\hline Numerical values & Verbal score \\
$<0.01$ & Negligible \\
$0.01-0.249$ & Low \\
$0.25-2.49$ & Moderate \\
$2.5-9.99$ & High \\
\hline $10-100$ & Very high \\
\hline
\end{tabular}

TABLE 9 Definitions of the verbal scores for probability and consequences

\begin{tabular}{|c|c|c|}
\hline Score & Probability & Consequences \\
\hline Negligible & $\begin{array}{l}\text { Rare, needs not to be } \\
\text { considered }\end{array}$ & $\begin{array}{l}\text { Insignificant, need not to be } \\
\text { considered }\end{array}$ \\
\hline Low & Rare, cannot be ruled out & $\begin{array}{l}\text { Insignificant, cannot be ruled } \\
\text { out }\end{array}$ \\
\hline Moderate & Happens sometimes & $\begin{array}{l}\text { Must be prepared for the } \\
\text { consequences }\end{array}$ \\
\hline High & Happens often & Consequences are serious \\
\hline Very high & $\begin{array}{l}\text { Happens almost } \\
\text { always }\end{array}$ & Consequences are very serious \\
\hline
\end{tabular}

Bluetongue, ASF and FMD. The consequence scores for disease release and exposure were then compared with the financial losses following an outbreak of a typical size (Figure 4). The financial losses (Bluetongue $€ 4.7 \mathrm{M}$, ASF $€ 10.5 \mathrm{M}$ and FMD €27.7 M) were retrieved from previously published risk assessments (Lyytikäinen et al., 2015). Schmallenberg, being a disease not controlled by the authorities, was considered to have zero consequences (multiplier effects, disease classification, menace and the zoonotic nature of the disease all giving a score of zero), and the financial losses for the society were assumed to be close to zero euros. However, Schmallenberg as a disease causes losses and social impacts to the producers that may lead to indirect financial losses for the society. Those consequences are not considered in NORA, as it is designed for assessing the risks of concern to the government.

\section{3 | Overall risk}

The probability of ASF introduction into Finland was assessed as high (0.196), the consequences were assessed as high (17.36), and the overall risk estimate was therefore high (3.4) when assessed with NORA. The results indicate the relative risk in comparison with other diseases.

\section{4 | DISCUSSION}

NORA was developed to assist the animal health authorities with risk management questions. It appears to be a good tool, corresponding well to the expectations. It provides a rapid estimate for the risk and is easy to use.

We used routes of ASF entry for the evaluation of disease spread. Routes were obtained mostly from the earlier risk profile on possible routes of entry into Finland for African swine fever (Oravainen et al., 2011) except for the air current/vector, feed or bedding materials and other traffic than animal transport vehicles, which were not considered when conducting the profile. Also, in the profile we mapped the possible routes and their volumes but we did not assess the concerning risks. A thorough assessment of the validity of the tool can only be made if ASF enters Finland. However, any calculated semiquantitative outcome for single or multiple pathogen risk assessments should be validated through an internal check with the qualitative information, through external validation against other approaches and through peer review.

We tested the method by applying four groups with different areas of expertise who tested the NORA tool with ASF as the assessed disease. The mean ranks for the overall probability of ASF entry did not differ between the groups. Thus, the method appears to give consistent results. However, the members of the research group are aware of the subjectivity involved in the method.

The results from the expert groups were compared. All routes except for air currents and/or vectors were assessed to exist by all four validation groups. These routes pose severe threats to the Finnish pig industry. Estonia and Russia are very important countries regarding the spread of disease to Finland due to heavy traffic and large numbers of passengers travelling between the countries. Products of animal origin, including sausages, are common souvenirs from Estonia and Russia. In addition, there are many Russian, Ukrainian and Estonian workers on Finnish farms. Finnish hunters have hunted 


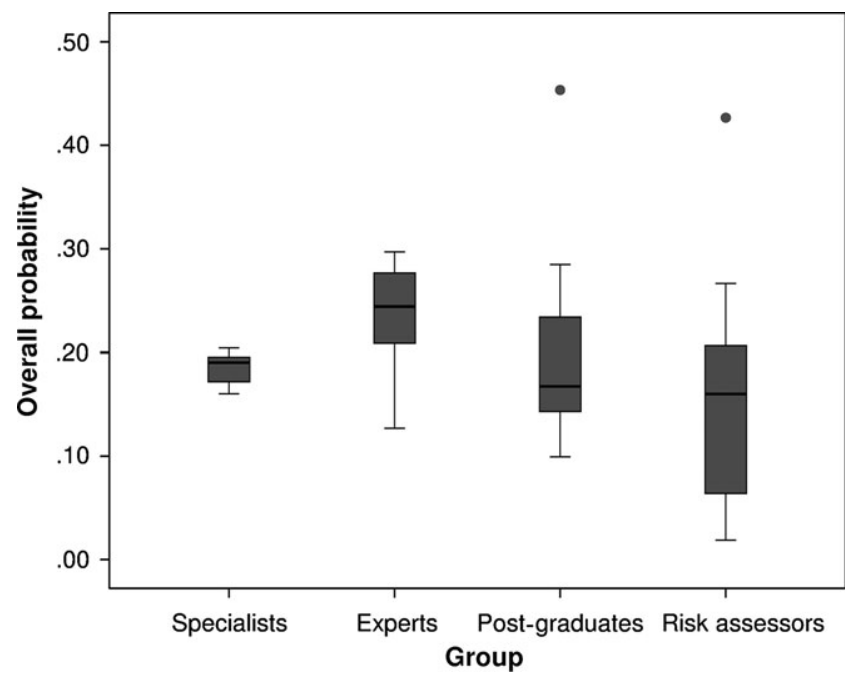

FIGURE 2 Overall probabilities for the entry of African swine fever estimated by the four validation groups (specialists, $n=5$; experts, $n=5$; postgraduates, $n=9$; and risk assessors, $n=12$ ). The interquartile range is the difference between the 75th and 25th percentiles and corresponds to the length of the box. The lines in the boxes represent the medians, the whiskers represent the minimum and maximum, unless there are values more than 1.5 times the interquartile range above 75th percentile, in which case it is the third quartile plus 1.5 times the interquartile range. Circles represent outliers that are $>2$ box lengths above the 75 th percentile

wild boars in the Baltic countries for many years and in large numbers.

The variation between the validation group residuals was significantly different for all other transmission routes except two: with animals and with feed and bedding materials. These might have been the easiest routes to evaluate, thus leading to a similar level of variation between the groups. It is easy to understand the effect of direct transmission with live animals and the low influence on spread posed by feed and bedding, even without thorough background knowledge of animal disease transmission. Transmission with air streams and/or vectors was a non-existent route according to all groups, leading to zero variation.
The amount of variation within the groups depended on the background of the group members. The group of veterinarians specializing in transmissible animal diseases were the most uniform in their answers. The animal health risk managers were the second most uniform, and the postgraduates and the risk assessors of food and plant health varied the most in their answers. Higher numbers of respondents diminished the confidence interval (variation), supporting the law of large numbers. With larger numbers of respondents in these groups, the variation would have decreased even more. The explanation for this might be that veterinarians involved with transmissible diseases hold the most information on the routes of entry for ASF, thus ending up with similar estimates. The postgraduates mostly studied companion animals and horses, and the risk assessors did not work with animal health issues at all.

The NORA scores were 6.7 for products of animal origin (including illegal imports), 3.6 for live animals, 1.2 for other goods and traffic, 1.1 for animal transport vehicles and 0.45 for wild boar. The route for products of animal origin (including illegal imports) has the highest score in the results, largely because there is a large volume of passengers between the Baltic countries and Finland. The Baltic countries and Finland are both part of the Schengen area, and therefore, there are no regular border or customs inspections.

The route with live animals, despite having the highest weight in the model and being the most easily transmitting route, is not as alarming as might be presumed, as Finland does not trade pigs or pig sperm with infected countries and Norway is the only country outside the EU that Finland imports pigs from. Overall, the level of animal trade is low. The small number of imported animals and germ cells as well as the limited volumes of other import pathways makes the probability of introduction lower than in many other countries, such as the UK (Defra, 2009).

Along with the official regulations, Finnish farmers follow many voluntary recommendations, as advised by the national animal health organization (ETT). The aim of ETT is to hinder transmissible animal disease introduction through trade and travel. Backyard pigs and keeping pigs outdoors are very uncommon in Finland. Wild boars might pose a risk to Finland, but the Finnish wild boar population is only about 1,500-2,000 animals (Ohto Salo, Finnish Wildlife Agency).
FIGURE 3 Mean scores of entry of African swine fever estimated by the four validation groups for the nine pathways: with animals (1), sperm and embryos (2), products of animal origin (3), passengers (4), wildlife (5), air streams and/or vectors (6), animal transport vehicles (7), feed and bedding materials (8) and other goods and traffic (9). [Colour figure can be viewed at wileyonlinelibrary.com]

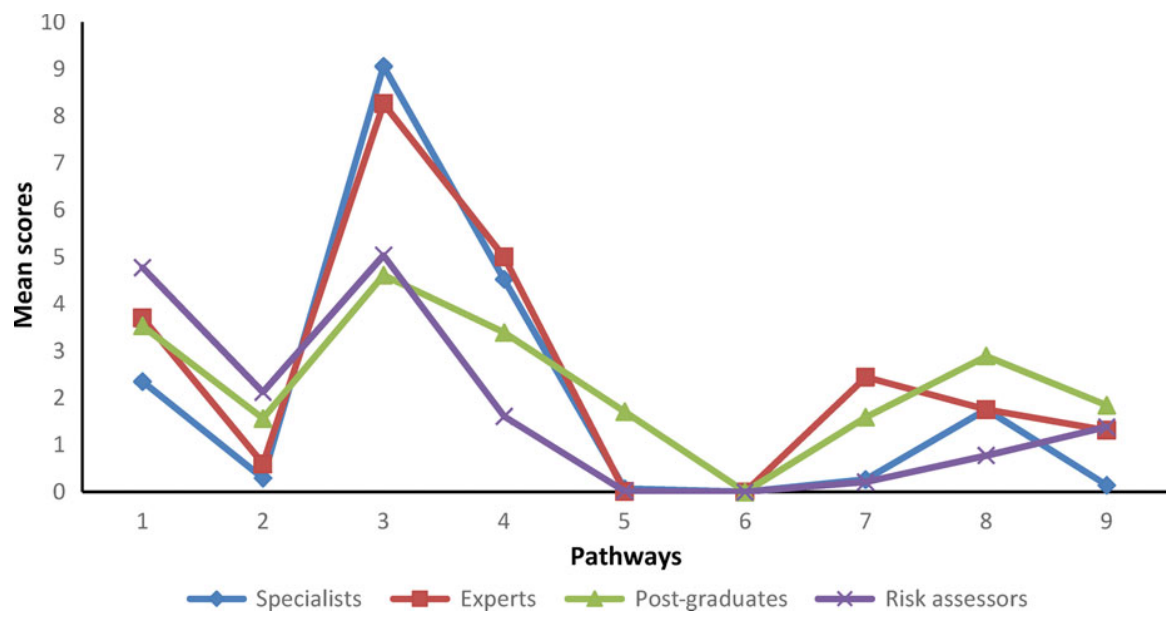




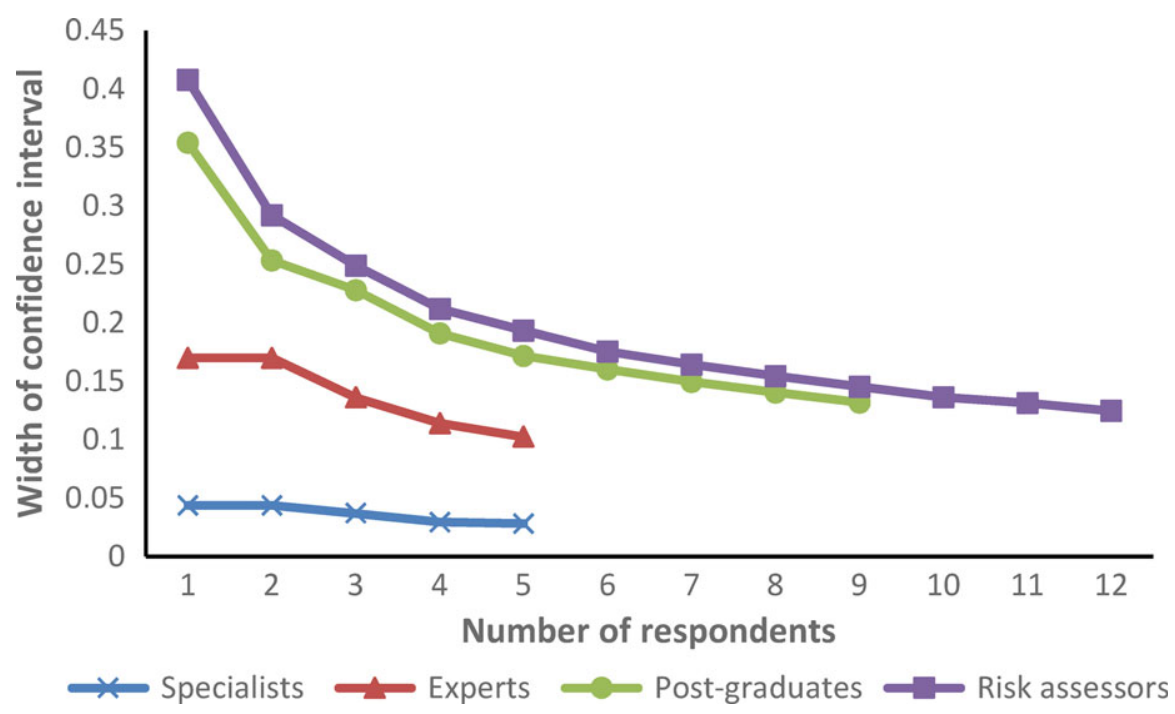

FIGURE 4 Effect of the number and background of experts on the simulated width of the $95 \%$ confidence interval of the mean value for the overall probability estimate of entry of African swine fever. [Colour figure can be viewed at wileyonlinelibrary.com]

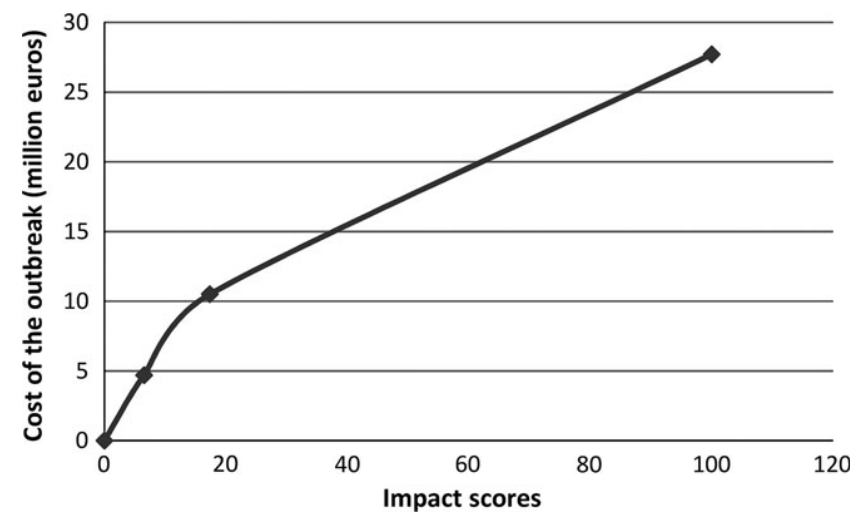

FIGURE 5 The association between the consequence scores for Schmallenberg (0), Bluetongue (6.54), ASF (17.36) and FMD (100) and the costs ( $€ 0, € 4.7 \mathrm{M}, € 10.5 \mathrm{M}$ and $€ 27.7 \mathrm{M}$, respectively) for an outbreak of a typical size in Finland

The density (less than 0.02 wild boar $/ \mathrm{km}^{2}, \mathrm{FAO}, 2010$ ) and movements of wild boar in Russia next to the Finnish border are low (Oravainen et al. 2011).

Our approach can be compared with several semiquantitative assessments of the risk of ASF introduction, including results achieved with the Pandora tool (Roelandt, Van der Stede, Dhondt, \& Koenen, 2015) and assessments by De la Torre et al. (2013), Mur et al. (2012), Costard et al. (2013) and Bosch et al. (2016). Roelandt et al. (2017) recently published their semiquantitative Pandora open source screening protocol with an assessment of the ASF risk to Belgium in early 2014. The results of Pandora and NORA cannot be compared directly, because they apply to different countries and the relevancy of pathways may vary. However, the Pandora framework is basically the same as that of NORA, with emergence and consequence scores that give an overall risk score as their product. Pandora considers entry and exposure separately and then aggregates them, whereas NORA gives a joint entry and exposure score. Roelandt et al. (2017) used European and Belgian experts and found the averaged emergence risk for ASF to be low, disease consequences high and the resulting multiplicative overall risk low. In contrast to this study, the Pandora entry module comprises only one question and one score. Nonetheless, they assessed that their entry simplification has not led to underestimation or overestimation of the risk of introduction as compared to other approaches from Mur et al. (2012) Mur et al. (2012) and Costard et al. (2013). Roelandt et al. (2017) concluded that hunting of wild boar populations, illegal import of local meat and wild boar movements are important risk factors. These pathways are also considered in the Finnish NORA model, but naturally their importance may vary due to geographical and operational differences between countries.

Mur et al. (2012) assessed the quantitative risk for ASF introduction into the EU by legal import of live pigs. They showed that legal trade of live pigs does not pose a high risk for ASFV introduction into the EU (only one outbreak in 192 years, on average). The results varied from each other when data from TRACES or EUROSTAT were used. Finland had a low risk for introduction, when EUROSTAT was used, taking into account import from Norway, the only country where pigs were imported from in 2010. Also, after 2010 Norway is the only country, outside the EU, from which pigs are imported to Finland.

Costard et al. (2013) presented a novel risk assessment framework for disease introduction into the EU through illegal importation of meat and meat products. They used wild boar suitable habitat as a proxy, not the number or density of wild boar. Finland has a lot of suitable habitat but a very low number of wild boar in the area close to the Russian border. The density of wild boar on the Russian side of the border is also low (Oravainen et al. 2011). According to the study of Costard et al. (2013) the herd size was used to assess the biosecurity level of farms. At that time Finland still had quite small herds, which despite their small size had high biosecurity standards (Sahlström, Virtanen, Kyyrö, \& Lyytikäinen, 2014). Even though low proxys (2/5), these may have led to Finland having a too high risk score. Costard et al. (2013) stated that the lack of detailed national data on both wild boar and non-high-biosecurity farms makes estimation of areas of interface between 
non-high-biosecurity farms and wild boar difficult, and is an important limitation of their study.

De la Torre et al. (2013) assessed the risk of African swine fever introduction into the EU by wild boar and updated the assessment in 2016 (Bosch et al., 2016). They concluded Finland to have the highest risk score (risk category 5 in 2013 and 4 in 2016) and that suitable wild boar habitat and outbreak density in the neighbouring countries are the two most important risk estimators. They state that their results help to identify countries where subnational analysis should be a high priority and that they had a relatively small set of input data. Indeed, Russia was given only one figure for density of domestic pig notifications (of ASF), and being such a large country, it should have been assessed in smaller areas, because those outbreaks are happening mostly in the southern parts of the country and in the Tver area. Also, the distance to the nearest wild boar and domestic pig notifications (for ASF) were given as $114.14 \mathrm{~km}$ and $128.03 \mathrm{~km}$ for Finland, respectively. Those distances must be from Estonian outbreaks; however, the Baltic Sea hinders the spread of the disease between these countries with wild boar.

Unlike the methods of, i.e., ECDC (2011) and Mur et al. (2014) that work on the European level, NORA was developed for Finnish risk assessment needs. Mur et al. (2014) assessed the relative release risk of ASF to $27 \mathrm{EU}$ countries. The relative risk to Finland was assessed to be $5 / 5$ with wild boar, $4 / 5$ with legal pigs, $3 / 5$ with transport-associated routes and 2/5 with illegal imports of pig products when each route was compared separately among $27 \mathrm{EU}$ countries. Our results cannot directly be compared with the results of Mur et al. (2014), because we have estimated the importance of these routes for the introduction risk for Finland. Moreover, NORA considers both release and exposure so the overall risk level should be lower than when only the release risk is considered. However, a slight discrepancy can be observed between the results of these two assessments. NORA considers the wild boar risk to be the least significant pathway for introduction to Finland, and Mur et al. (2014) considers that wild boar release risk to Finland is in the highest category within the EU. Also the estimated risk of live pigs might reveal some discrepancy between studies. Mur et al. (2014) used EU databases and concluded that some of them were not as complete and detailed as the national databases and that this may have resulted in under- or overestimations of risk scores. Particularly, information on wild boar density and backyard pig production was limited and could have affected the model results. We think that the low number of wild boar in Finland, the absence of backyard pigs and the fact that Finland imports pigs from outside EU only from Norway, are actually facts that if not taken into consideration, may lead to false interpretations and introduce country-related bias into the relative risk estimates of pathways in EU.

Finland was given a very high (5/5) relative risk for ASFV introduction into the EU by waste from international ships and medium risk (2/5) for waste from international flights. The latter was described in the earlier risk profile we conducted, along with waste from the international railways (Oravainen et al., 2011). The model by Mur et al. (2012) did not provide probabilities, but compared the relative risk between EU countries based on the risk factors evaluated, i.e., a high value on the model results does not imply an absolute high level of risk. Furthermore, the model estimated only the risk of release of potential ASFV-contaminated material/transports, but did not consider the subsequent exposure. The only transportassociated route included in NORA is the returning trucks. Consideration of risk associated with waste from airplanes and ships could benefit the NORA tool. However, the inclusion of exposure risk would reduce the probability of these routes considerably. The probability of exposure of pigs and wild boar to this waste is low, if it is handled according to recommendations and good biosecurity practices are followed. For this reason the present version of NORA excludes international waste as a potential route to infect animals in Finland.

Even though national data were available to us for many pathways, some pathways needed to be answered without detailed information of the volumes (professionals from abroad working with animals, feed, bedding materials and other goods). Particularly, volume of illegal import of animals, sperm and products of animal origin (POAO) are almost impossible to know in detail, even though POAO were assessed by the number of personal consignments confiscated during the earlier year. However, national data sources seem to be more accurate than EU level databases in general.

For easy use of NORA, we wrote a guidebook in Finnish. It includes a summary, a detailed description of the tool and its mathematical foundations, a quick guide for using NORA and appendices with background information to be used when answering the NORA questions. The appendices include the following: the classification of animal diseases in Finnish legislation, a flow chart for animal disease release and exposure risk, imports of animals, sperm and embryos, imports of products of animal origin from third countries, the main trading partners of Finland, the numbers of passengers from different countries, the numbers of foreign workers on Finnish farms and a list of consulted experts.

The consequences are assessed with yes/no questions that are unaffected by opinions. Therefore, the consequence section of the tool was not assessed by the validation groups. The association of the scores for consequences of the four diseases assessed and the predicted costs of the disease outbreaks were almost linear, which indicates that the scores closely approximate the importance of the disease event. This approach of not using the experts in consequence assessment is different from, for instance, Dufour et al. (2011). They had scores between zero and three assigned for the three items considered for animal health and three for human health. Their four-level scale is a subject for expert assessments.

Estimates for probability, consequences and overall risks in NORA are obtained both in numbers and as verbal scores. This can be an asset: some people merely like to see numbers, while others need to have a verbal score to feel comfortable with the answers. NORA serves both types of user and enables easy translation between them. However, one should bear in mind that the same verbal statement can actually mean a different risk level for different people. For instance, the level that means an acceptable risk for one 
person might be a level that requires immediate intervention according to another. Therefore, definitions of the verbal scores for probability and impact are included in NORA.

Uncertainty is not built into the NORA framework. It is possible to estimate it by doing a combination of several point wise estimations, as was carried out when validating the tool in this study. However, by adding a Monte Carlo simulation option into NORA, it would be possible to include uncertainty in a more coherent way. For the practical use of NORA, namely rapid risk assessment, simulation is rarely required.

NORA is a semiquantitative tool, and thus, also the interpretation of the results needs to be both verbal and numerical. Although there are qualitative verbal definitions, the location within the numerical class should be taken into account. The estimated risk might be close to a limit between two classes and is therefore relatively sensitive to small changes in input values.

The weights for different disease transmission routes and subfactors of consequences were assigned by the research group. Weights for the nine different pathways were ranked so that the pathways with the same significance were given the same rank. One hundred points were divided between the pathways according to their ranks. This is a fairly subjective and robust method, even though based on published literature. Therefore, when assessing a new disease, these weights should be assessed with the help of disease-specific experts.

The weighting for the subfactors for consequences was assigned by giving FMD the highest score (100) and then scaling down the other three diseases (BT, ASF and Schmallenberg) to correspond to the estimated mean expenses of the disease epidemic. Therefore, weights for consequence factors are related to the financial losses of the epidemics and are not as subjective as weights for pathways.

NORA is a rapid tool to use, which makes it easy to run it again whenever new information becomes available on a changing situation concerning disease spread. However, using experts in animal health risk assessments in Finland can be problematic, because the number and availability of experts is low. The results demonstrate that depending on the experts used, a few well-picked experts are sufficient to obtain a reasonable result. A small meeting or a summary with an update on the disease status and the situation at hand may be enough before the assessment. For example, in the case of new evidence of illegal imports of pigs or pork from the infected areas, updating the assessment with NORA would be useful.

We will continue using NORA also for other diseases and scenarios. Each disease needs to be validated within the research group and a group of disease-specific experts. ASF can act as a benchmark and form a basis on which to derive probabilities of entry and exposure for other diseases.

\section{ACKNOWLEDGEMENTS}

The authors wish to thank Prof. Gordon Copp, Dr Edmund Peeler and Dr Lorenzo Vilizzi for sharing an open source version of the non-native freshwater fish risk identification toolkit (FISK), which we used to gain ideas for an easy interface for NORA that will probably follow. We also thank all the respondents in our validation groups and Dr Kitty Schulman for valuable comments.

\section{REFERENCES}

Bosch, J., Rodríguez, A., Iglesias, I., Muñoz, M. J., Jurado, C., Sánchez-Vizcaíno, J. M., \& de la Torre, A. (2016). Update on the risk of introduction of African swine fever by wild boar into disease-free European Union Countries. Transboundary and Emerging Diseases, doi:10.1111/ tbed.12527

Costard, S., Jones, B. A., Martinez-Lopez, B., Mur, L., de la Torre, A., Martinez, M., ... Wieland, B. (2013). Introduction of African swine fever into the European Union through illegal importation of pork and pork products. PLoS ONE, 8, e61104.

De la Torre, A., Bosch, J., Iglesias, I., Munoz, M. J., Mur, L., MartinezLopez, B., ... Sanchez-Vizcaino, J. M. (2013). Assessing the risk of African swine fever introduction into the European Union by wild boar. Transboundary and Emerging Diseases, 62, 272-279.

Defra (Department for Environment, Food and Rural Affairs) (2009). Exotic animal disease risk pathways 6 countermeasures. Final Report. Retrieved from https://www.gov.uk/government/uploads/system/ uploads/attachment_data/file/69427/pb13567-risk-pathways-counte rmeasures-100310.pdf. Accessed April 7, 2016.

Dufour, B., Plee, L., Moutou, F., Boisseleau, D., Chartier, C., Durand, B. ... Toma, B. (2011). A qualitative risk assessment methodology for scientific expert panels. Revue Scientifique et Technique, 30(3), 673681.

ECDC (European Centre for Disease Prevention and Control) (2011) Operational guidance on rapid risk assessment methodology. Stockholm: ECDC.

EFSA (2012). Scientific opinion on risk assessment terminology. EFSA Journal, 10(5), 2664. [43 pp.] doi:10.2903/j.efsa.2012.2664 Retrieved from http://onlinelibrary.wiley.com/doi/10.2903/j.efsa.2012.2664/f ull. Accessed September 7, 2016.

EFSA, AHAW Panel (2010). Scientific opinion on African swine fever EFSA Journal, 8(3), 149.

EFSA, AHAW Panel (2014). Scientific opinion on African swine fever. EFSA Journal, 12(4), 77.

FAO (2010). FAO takes a close look at the pig sector in Eastern Europe. Rome: EMPRES WATCH. Retrieved from http://www.fao.org/docre p/012/ak755e/ak755e00.pdf. Accessed March 10, 2016.

Food and Agricultural Organisation (FAO) (2013). African swine fever in the Russian Federation: Risk factors for Europe and beyond. Empres Watch, 28, 1-14.

Gogin, A., Gerasimov, V., Malogolovkin, A., \& Kolbasov, D. (2013). African swine fever in the North Caucasus region and the Russian Federation in years 2007-2012. Virus Research, 173, 198-203.

Lyytikäinen, T., Niemi, J. K., Sahlström, L., Virtanen, T., Rintakoski, S., Kyyrö, J., ... Lehtonen, H. (2015). The effects of structural change in agriculture on the spread of animal disease in Finland. Evira Research Reports 3/2015.

Morgan, D., Kirkbride, H., Hewitt, K., Said, B., \& Walsh, A. L. (2009). Assessing the risk from emerging infections. Epidemiology and Infection, 137, 1521-1530.

Moutou, F., Dufour, B., \& Ivanov, Y. (2001). A qualitative assessment of the risk of introducing foot and mouth disease into Russia and Europe from Georgia, Armenia and Azerbaijan. Revue Scientifique et Technique, 20(3), 723-730.

Mur, L., Martínez-López, B., Costard, S., de la Torre, A., Jones, B. A., Martínez, M., ... Wieland, B. (2014). Modular framework to assess the risk of African swine fever virus entry into the European Union. BMC Veterinary Research, 10, 145.

Mur, L., Martinez-Lopez, B., Martinez-Aviles, M., Costard, S., Wieland, B. Pfeiffer, D. U., \& Sanchez-Vizcaino, J. M. (2012). Quantitative risk 
assessment for the introduction of African swine fever virus into the European Union by legal import of live pigs. Transboundary and Emerging Diseases, 59, 134-144.

Mur, L., Martínez-López, B., \& Sánchez-Vizcaíno, J. M. (2012). Risk of African swine fever introduction into the European Union through transport-associated routes: Returning trucks and waste from international ships and planes. BMC Veterinary Research, 8, 149.

Oravainen, J., Sahlström, L., \& Lyytikäinen, T. (2011). Possible routes of entry into the country for african swine fever - Risk profilie. Evira Research Reports 5/2011, 1-66.

Palmer, S., Brown, D., \& Morgan, D. (2005). Early qualitative risk assessment of the emerging zoonotic potential of animal diseases. BMJ, 331, 1256-1260.

Roberts, H., Carbon, M., Hartley, M., \& Sabirovic, M. (2011). Assessing the risk of disease introduction in imports. Veterinary Record, 30, 447-448.

Roelandt, S., Van der Stede, Y., Dhondt, B., \& Koenen, F. (2017). The assessment of African swine fever virus risk to Belgium early 2014, using the quick and semiquantitative Pandora screening protocol. Transboundary and Emerging Diseases, 64, 237-249.

Sahlström, L., Virtanen, T., Kyyrö, J., \& Lyytikäinen, T. (2014). Biosecurity on Finnish cattle, pig and sheep farms - results from a questionnaire. Preventive Veterinary Medicine, 117(1), 59-67.

Sánchez-Vizcaíno, J. M., Mur, L., Gomez-Villamandos, J. C., \& Carrasco, L. (2015). An update on the epidemiology and pathology of African swine fever infectious disease: Review article. Journal of Comparative Pathology, 152, 9-21.

Sánchez-Vizcaíno, J. M., Mur, L., \& Martínez-López, B. (2013). African swine fever (ASF): Five years around Europe. Veterinary Microbiology, $165,45-50$.
Sánchez-Vizcaíno, J. M., Mur, L., Sánchez-Matamoros, A., \& MartínezLópez, B. (2014). African Swine Fever: New challenges and measures to prevent its spread. 82nd General Session World Assembly. Paris: OIE, 25-30 May 2014.

WHO (2012). Rapid risk assessment of acute public health events. Retrieved from http://apps.who.int/iris/bitstream/10665/70810/1/ WHO_HSE_GAR_ARO_2012.1_eng.pdf. Accessed March 10, 2016.

Wilson, R., \& Crouch, E. A. C. (2001). Risk-benefit analysis. 2nd Edn. Distributed by Harvard University Press. Copyright 2001: The President and Fellows of Harvard College. p. 9.

World Organisation for Animal Health (OIE) (2016). WAHID: World animal health information database. Retrieved from http://www.oie.int/wa his_2/public/wahid.php/Wahidhome/Home. Accessed September 14, 2016.

World Organization for Animal Health (OIE) (2011). Terrestrial animal health code, Chapter 2.1. In: Import risk analysis, 20th Ed. Paris: OIE. Retrieved from www.oie.int/doc/ged/D10905.pdf. Accessed October 7, 2016.

How to cite this article: Kyyrö J, Sahlström L, Lyytikäinen T. Assessment of the risk of African swine fever introduction into Finland using NORA — a rapid tool for semiquantitative assessment of the risk. Transbound Emerg Dis. 2017;64:21132125. https://doi.org/10.1111/tbed.12634 